\title{
Obtenção de espumas flexíveis de poliuretano com celulose de Pinus elliottii
}

\section{Flexible polyurethane foams filled with Pinnus elliotti cellulose}

\author{
Vinícius de Macedo*, Matheus Vinicius Gregory Zimmermmann², Letícia Scherer Koester³, \\ Lisete Cristine Scienza² e Ademir José Zattera ${ }^{1}$
}

\author{
${ }^{1}$ Laboratório de Polímeros, Centro de Ciências Exatas e Tecnologias, Universidade de Caxias do Sul - \\ UCS, Caxias do Sul, RS, Brasil \\ ${ }^{2}$ Departamento de Materiais, Universidade Federal do Rio Grande do Sul - UFRGS, Porto Alegre, RS, \\ Brasil \\ ${ }^{3}$ Faculdade de Farmácia, Universidade Federal do Rio Grande do Sul - UFRGS, Porto Alegre, RS, Brasil \\ *vinicius.demacedo@gmail.com
}

\begin{abstract}
Resumo
Neste trabalho foram desenvolvidas espumas flexíveis de poliuretano com a adição de celulose de Pinus nas concentrações de 0,$5 ; 1$ e $2 \%(\mathrm{~m} / \mathrm{m})$. A celulose foi submetida ao processo de fibrilação mecânica e posterior secagem por aspersão (spray dry) sendo caracterizada quanto a sua morfologia por MEV e MET. As espumas foram produzidas pelo método de batelada (one-shot) com a adição e mistura da fibra junto ao poliol. As espumas foram caracterizadas por MEV, densidade aparente e resistência à compressão. Os principais resultados indicam que a fibrilação mecânica promove a obtenção de fibras em escala nanométrica, porém durante a secagem, ocorre aglomeração ocasionando aumento para escala micrométrica. As propriedades mecânicas da espuma obtiveram acréscimos de 40 e $50 \%$ na resistência à compressão com a adição de 0,5 e $1 \%$ de celulose, respectivamente, evidenciando seu potencial como aditivo alternativo para o desenvolvimento de espumas de poliuretano.
\end{abstract}

Palavras-chave: celulose, espuma, fibrilação, poliuretano, spray dry.

\begin{abstract}
In this work, flexible polyurethane foams were developed with the addition of Pinus cellulose at concentrations of 0.5; 1 and $2 \mathrm{wt} \%$. The cellulose was subjected to mechanical fibrillation process and subsequent dried by spray dry process. It was characterized morphologically by SEM and TEM. The foams were produced by one-shot method by adding and mixing the fiber with the polyol. The foams were characterized by SEM, apparent density and compression strength. The main results indicate that the mechanical fibrillation promotes the obtainment of nanoscale fibers, but during the drying process, agglomeration occurs causing an increase reaching the micrometer scale. The mechanical properties of the foam increased by 40 and $50 \%$ in compressive strength with the addition of $0.5 \%$ and $1 \%$ of cellulose, respectively, showing its potential as an alternative additive for the development of polyurethane foams.
\end{abstract}

Keywords: cellulose, foam, fibrillation, polyurethane, spray-dry.

\section{Introdução}

Poliuretanos (PU) constituem uma classe de polímeros que possuem grande versatilidade e demanda industrial. São formados basicamente por ligações uretânicas (-NH-CO-O-), e pequenos ajustes em sua formulação permitem a obtenção de materiais específicos com diferentes propriedades para aplicações como revestimentos, adesivos e compósitos, entre outros $^{[1-3]}$. Espumas de PU podem ser classificadas como rígidas ou flexíveis, ou ainda, espumas de células abertas, quando as paredes de suas células são rompidas permitindo sua interligação por meio de poros, ou células fechadas, quando não há comunicação entre as células e o gás formado para expansão fica aprisionado em seu interior ${ }^{[1,4]}$. Entre as aplicações mais comuns de espumas flexíveis de PU, destacam-se: bancos de automóveis, colchões, travesseiros, móveis, embalagens, calçados, etc., por possuírem boa capacidade de amortecimento de impacto, flexibilidade, durabilidade e facilidade de moldagem entre outros ${ }^{[5-9]}$.

A polimerização de um diisocianato com reagentes que possuem hidrogênios ativos, tais como os diois (poliol), para formar uma rede tridimensional poliuretânica que juntamente com a reação de produção de um gás (reação entre isocianato 
e água produz gás carbônico) são os principais responsáveis pela formação de espumas de PU, sendo que outros reagentes são utilizados, tais como surfactantes, catalisadores e agentes de expansão auxiliares ${ }^{[1,3,6,7,10]}$. Quanto ao processo de produção de espumas de PU, elas podem ser obtidas pelo processo de batelada, conhecido como one-shot, no qual os reagentes são adicionados sequencialmente e agitados vigorosamente em uma única etapa para serem posteriormente descarregados em um molde de formato específico ${ }^{[6]}$.

Grande parte das propriedades específicas de uma espuma (densidade, resistência à compressão e rigidez) pode ser alterada pela adição de cargas, tais como: sulfato de bário, carbonato de cálcio ou por reagentes químicos de origem não renovável, como derivados do petróleo ${ }^{[3,7,11]}$. O interesse no uso de fibras vegetais cresceu significativamente nos últimos anos, ampliando suas aplicações no uso como agentes de reforços em materiais poliméricos devido, principalmente, ao seu grande potencial na substituição de fibras inorgânicas, como a fibra de vidro e carbonato de cálcio $^{[12]}$. A vantagem do uso de cargas de origem vegetal em materiais poliuretânicos consiste no fato de que, além de serem de origem renovável, possuem grupos hidroxila em sua superfície que podem formar ligações de hidrogênio com o grupo isocianato do $\mathrm{PU}$, tornando as fibras celulósicas um material quimicamente compatível em composições poliuretânicas ${ }^{[13,14]}$

A diminuição do tamanho da fibra de celulose pelo processo top-down (da escala micro até nano) ocorre com a fibrilação da parede celular, rompendo os emaranhados de fibras e liberando as nanofibras. Métodos como tratamento mecânico, térmico, químico e biológico permitem a obtenção de diferentes tipos de nanofibra, dependendo da matéria-prima e do pré-tratamento utilizado ${ }^{[15]}$. Nanofibra é um termo geral que classifica a celulose que possui pelo menos uma de suas dimensões em escala nanométrica ${ }^{[16]}$.

Um dos tipos de nanofibra, a celulose nanofibrilada (NFC), também é chamada de celulose microfibrilada, microfibrila, nanofibras de celulose, celulose nanofibrilar ou microfibras, são agregados de fibras longas e flexíveis compostas de regiões amorfas e cristalinas com diâmetro de $10 \mathrm{a} 100 \mathrm{~nm}$ e com comprimento de vários micrometros, possuem elevada área superficial e elevada razão de aspecto $^{[16-20]}$. Este tipo de nanofibra é obtido, geralmente, por processo mecânico, sendo que outros processos auxiliares podem ser empregados, tais como processo enzimático, oxidação mediada por N-oxil-2,2,6,6-tetrametilpiperidina (TEMPO), carboximetilação ou acetilação ${ }^{[19,21]}$. O processo mecânico com o uso de moagem por friç̧ão ultrafina é um método de uma única etapa e de alto rendimento, no entanto, possui alto consumo energético para sua operação e é realizado com suspensões aquosas celulósicas de $1 \%$ a $3 \%(\mathrm{~m} / \mathrm{m})^{[18,22-24]}$. Um equipamento utilizado para obtenção de NFC é o moinho fibrilador de moagem ultrafina e seu funcionamento é baseado na passagem da suspensão celulósica entre pedras de moagem, enquanto que uma das pedras é estacionária, a outra é mantida a rotações entre 1500 e $3000 \mathrm{rpm}$ e o material a ser moído é submetido a forças cisalhantes de corte que causam o rompimento da estrutura individualizando as fibras, obtendo $\mathrm{NFC}^{[17-22,25]}$.
Para utilização da NFC em materiais poliméricos, como ela geralmente é processada como uma suspensão aquosa, é necessário realizar a secagem dessa fibra celulósica. A escolha do método de secagem da nanocelulose é um desafio na área tecnológica devido a sua natureza hidrofílica, com a necessidade de diminuição de etapas de processo para redução de custos além de garantir que sejam mantidas as características nanométricas das fibras, evitando a sua aglomeração. Devido à fibrilação, a superfície da celulose fica exposta a uma maior concentração de grupos hidroxilas, que tendem a formar ligações de hidrogênio com as fibras vizinhas, difícultando a dispersão da celulose e formando emaranhados parcialmente reversíveis ou irreversíveis ${ }^{[1,26-28]}$.

Dentre as várias técnicas conhecidas para secagem, a realizada por aspersão (spray dry) é uma das mais comumente utilizadas na indústria para secagem de suspensões aquosas. Esta técnica permite rápida transferência de calor e massa de modo contínuo, além de eliminar etapas posteriores de processamento para a separação de aglomerados (moagem). Neste equipamento o fluido é disperso na câmara de secagem pelo atomizador produzindo gotículas que possuem grande área superficial. Estas gotículas entram em contato com uma corrente de ar aquecido, havendo transferência de calor e massa para evaporação do solvente. Com o aumento da área superficial, o solvente que envolve a partícula evapora rapidamente e o líquido interno desta difunde-se para a sua superfície, secando-a e formando partículas sólidas ${ }^{[29,30]}$. Alguns parâmetros de operação que influenciam no processo são: temperatura do ar de entrada, fluxo de entrada do material, taxa de evaporação do líquido e separação das partículas secas do gás ${ }^{[26,29]}$.

Frente ao exposto, o objetivo deste trabalho é desenvolver uma espuma poliuretânica e avaliar a influência da adição de nanocelulose de Pinus obtida através de processo mecânico e secagem por spray dry em suas propriedades físicas, morfológicas e mecânicas.

\section{Materiais e Métodos}

Os materiais utilizados para obtenção das espumas poliuretânicas foram Poliol Voranol WL 4010, diisocianato de tolueno (TDI) Voranate T-80 e cloreto de metileno, fornecidos pela Dow Brasil Sudeste Industrial Ltda.; Niax silicone L-595, fornecido pela Momentive Performance Materials Inc.; amina terciária Dabco 2033 Catalyst, fornecida pela Air Products Brasil Ltda. e octoato de estanho Kosmos 29, fornecido pela Evonik S.A.

A celulose de origem da madeira de Pinus elliotti (CP), não branqueada, foi fornecida pela empresa Trombini S/A de Caxias do Sul, Brasil, na forma de folhas com teor de umidade de aproximadamente $65 \%$.

\subsection{Moagem da celulose por fricção ultrafina}

Antes de sua utilização, as folhas de celulose foram secas em estufa durante $8 \mathrm{~h} \mathrm{a} 70{ }^{\circ} \mathrm{C}$ para evitar degradação microbiológica. Após secagem em estufa, a $\mathrm{CP}$ foi submetida a uma pré-moagem em um moinho Marconi modelo MA580 com peneira de diâmetro $\emptyset$ de $2 \mathrm{~mm}$ para separação das fibras. Após, a celulose foi submetida a um processo de fibrilação por fricção ultrafina utilizando o moinho Super 
Masscolloider MKCA6-2 marca Masuko®. A fibrilação foi realizada com uma suspensão de 3\% de fibras de celulose em água destilada e a moagem foi realizada por um período de 5 horas. A pedra rotatória de moagem foi mantida a uma rotação de $2500 \mathrm{rpm}$ e foi mantido o "modo contato" entre a pedra estacionária e a rotativa durante operação conforme Figura 1. Utilizou-se uma motobomba centrífuga para promover a recirculação da suspensão de celulose e água pelo moinho. Após moagem, o produto desta operação foi uma suspensão celulósica de $2,23 \%(\mathrm{~m} / \mathrm{m})$ de celulose.

\subsection{Secagem da celulose por spray dry}

A secagem da suspensão foi realizada por processo de aspersão (spray dry) utilizando equipamento P6.3 GEA Niro Spray dryer. Para sua operação foram mantidas as temperaturas de entrada e saída em $140{ }^{\circ} \mathrm{C}$ e $100{ }^{\circ} \mathrm{C}$, respectivamente, e velocidade de rotação do atomizador em $12800 \mathrm{rpm}$. O fluxograma básico envolvendo as etapas de processamento realizado na celulose antes da obtenção das espumas pode ser visualizado na Figura 2.

\subsection{Método de obtenção das espumas e compósitos expandidos}

A Tabela 1 apresenta a formulação utilizada para obtenção das espumas de PU. A concentração de celulose utilizada foi de $0,5 \% ; 1 \%$ e $2 \%$ sobre a massa total de reagentes. As espumas foram produzidas por batelada (one-shot), segundo um procedimento descrito na literatura ${ }^{[4,7,11,31]}$. Inicialmente, o poliol e as fibras foram misturados utilizando um misturador Fisaton modelo 715 com velocidade de rotação de $2500 \mathrm{rpm}$ até a completa dispersão por um período de aproximadamente $1 \mathrm{~min}$. Após, água, amina e silicone foram adicionados e agitados por $40 \mathrm{~s}$. Na sequência o octoato de estanho foi adicionado e misturado por $20 \mathrm{~s}$, seguido de um período de repouso de $20 \mathrm{~s}$. O TDI foi adicionado à mistura sob agitação vigorosa por 5 a $7 \mathrm{~s}$, sendo posteriormente descarregado em um molde de madeira para expansão da espuma. Após o período de cura de 48 horas as espumas foram cortadas com o uso de serra fita e submetidas aos ensaios de caracterização. Na espuma sem adição de celulose não foi necessária a primeira etapa descrita neste método.

\subsection{Caracterizações}

A morfologia da celulose e das espumas foi avaliada por microscopia eletrônica de varredura (MEV), realizada em um microscópio eletrônico marca Shimadzu modelo Superscan SS-500. As amostras foram recobertas com uma fina camada de ouro antes de serem analisadas. Foi utilizada uma tensão de aceleração de $5 \mathrm{kV}$ a $15 \mathrm{kV}$.

A morfologia da suspensão de celulose após o processo de moagem foi realizada por microscopia eletrônica de transmissão (MET) em um microscópio eletrônico Jeol Jem 2010. A amostra foi preparada com uma diluição de 0,12 g. $\mathrm{L}^{-1}$ de celulose e utilizou-se uma gota desta diluição em um suporte de cobre de 400 mesh revestido com filme de formvar. Em seguida uma gota de uma solução contraste de acetato de uranila foi adicionada à amostra. O sistema

Tabela 1. Formulação padrão utilizada para obtenção das espumas poliuretânicas.

\begin{tabular}{lcccc}
\hline \multicolumn{1}{c}{ Material } & EPU & EPU0,5 & EPU1 & EPU2 \\
\hline Poliol & $100 \mathrm{~g}$ & $100 \mathrm{~g}$ & $100 \mathrm{~g}$ & $100 \mathrm{~g}$ \\
TDI & $51,5 \mathrm{~g}$ & $51,5 \mathrm{~g}$ & $51,5 \mathrm{~g}$ & $51,5 \mathrm{~g}$ \\
Água & $4,2 \mathrm{~g}$ & $4,2 \mathrm{~g}$ & $4,2 \mathrm{~g}$ & $4,2 \mathrm{~g}$ \\
Silicone & $0,8 \mathrm{~g}$ & $0,8 \mathrm{~g}$ & $0,8 \mathrm{~g}$ & $0,8 \mathrm{~g}$ \\
Amina & $0,12 \mathrm{~g}$ & $0,12 \mathrm{~g}$ & $0,12 \mathrm{~g}$ & $0,12 \mathrm{~g}$ \\
Octoato de Estanho & $0,18 \mathrm{~g}$ & $0,18 \mathrm{~g}$ & $0,18 \mathrm{~g}$ & $0,18 \mathrm{~g}$ \\
Cloreto de Metileno & $12 \mathrm{~g}$ & $12 \mathrm{~g}$ & $12 \mathrm{~g}$ & $12 \mathrm{~g}$ \\
Celulose de Pinus & - & $0,844 \mathrm{~g}$ & $1,688 \mathrm{~g}$ & $3,376 \mathrm{~g}$ \\
\hline
\end{tabular}

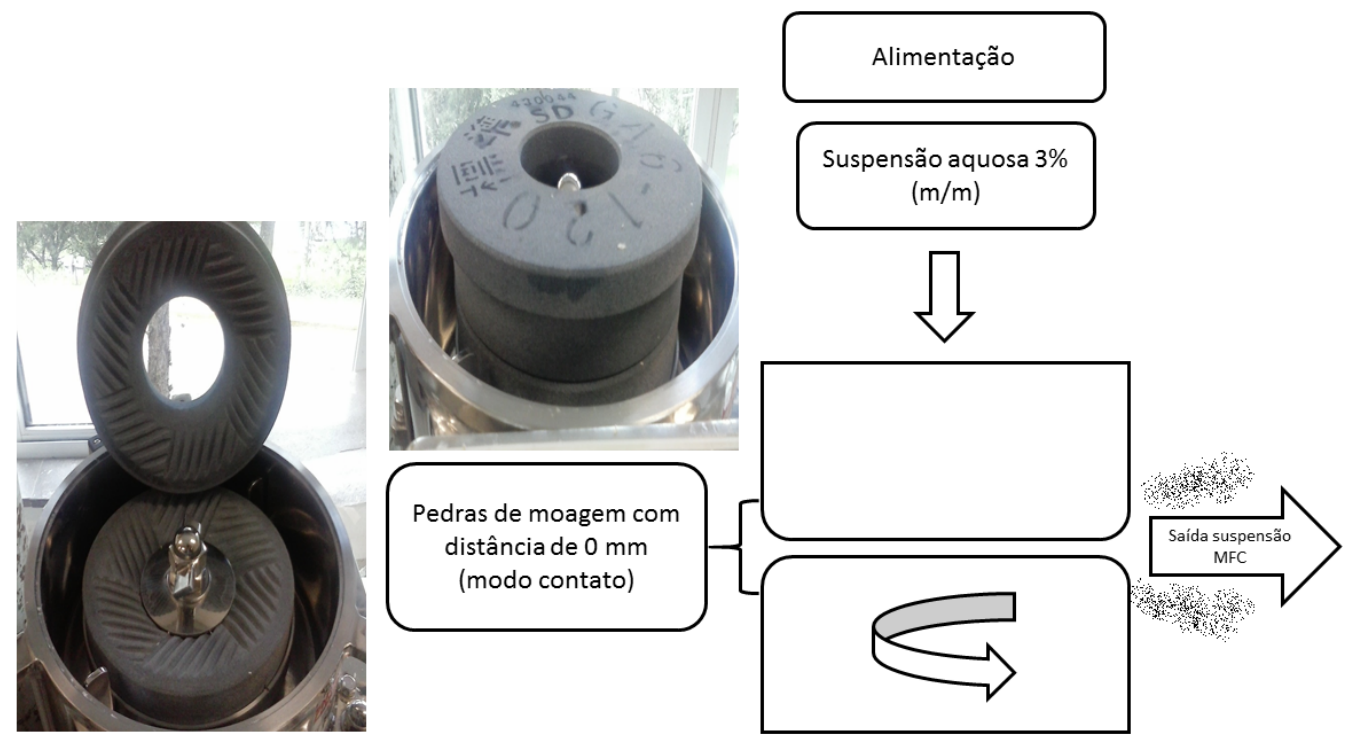

Figura 1. Esquema de funcionamento das pedras de moagem de moinho fibrilador Masuko. 


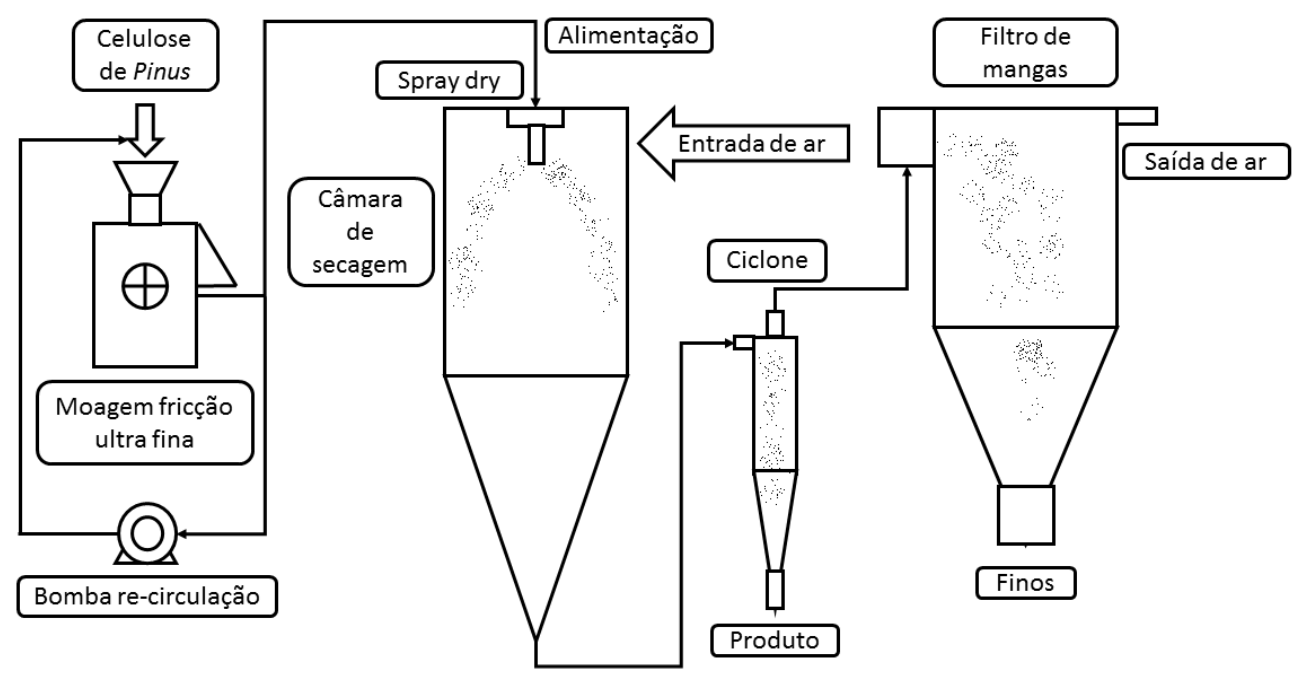

Figura 2. Etapas do processamento realizado na celulose de Pinus para posterior utilização nas espumas de PU.

celulose/uranila foi mantido em dessecador durante 24 horas antes da análise.

A densidade aparente das amostras foi realizada conforme norma ASTM D3574-11. O método consiste em determinar a massa de uma amostra com as dimensões de $50 \times 50 \times 25 \mathrm{~mm}$. A propriedade foi calculada pela razão entre a massa e o volume da amostra. Foram utilizadas 5 amostras de cada formulação.

O cálculo da densidade de células foi realizado por meio da análise de três micrografias de MEV conforme Equação 1, que relaciona a quantidade de células presentes em $1 \mathrm{~cm}^{3}$ de amostra segundo literatura ${ }^{[4,5,32]}$.

$$
\mathrm{D}_{\mathrm{c}}=\left(\frac{\mathrm{n} \cdot \mathrm{M}^{2}}{\mathrm{~A}}\right)^{\frac{3}{2}}
$$

Onde: $\mathrm{D}_{\mathrm{c}}=$ Densidade de células $\left(\right.$ células. $\left.\mathrm{cm}^{-3}\right), \mathrm{n}=$ quantidade de células presentes na micrografia de $\mathrm{MEV}, \mathrm{M}=$ fator de magnitude da micrografia de $\mathrm{MEV}, \mathrm{A}=$ área da micrografia de $\operatorname{MEV}\left(\mathrm{cm}^{2}\right)$.

O tamanho dos poros das espumas foi calculado através de micrografias de MEV com a utilização do software ImageJ empregando a ferramenta diâmetro Feret. A dimensão das fibras foi calculada empregando a ferramenta measure.

Os ensaios de resistência à compressão das espumas foram realizados em triplicata em um equipamento universal de ensaios EMIC modelo DL 2000. Corpos de prova de $50 \times 50 \times 25$ mm e velocidade de compressão de $50 \mathrm{~mm}^{\mathrm{min}} \mathrm{m}^{-1}$ foram utilizados para medir a tensão necessária para reduzir a espessura do corpo de prova em até $50 \%$ da sua espessura inicial, conforme a norma ASTM D3574-11.

\section{Resultados e Discussão}

A Figura 3 apresenta as micrografias obtidas por MEV. Para obtenção da micrografia da Figura 3 b foi necessário secar um filme de celulose em estufa e rasgá-lo posteriormente.
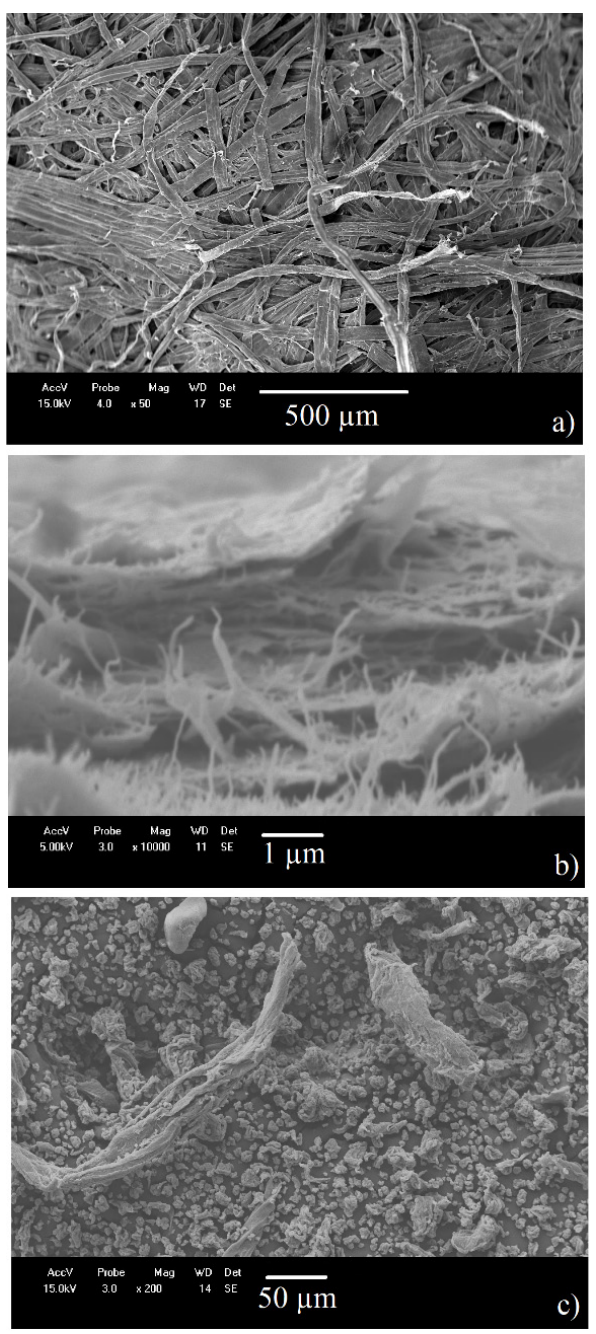

Figura 3. Micrografias de MEV das amostras de celulose de Pinus: (a) antes do processamento; (b) após 5 horas de moagem e secagem em estufa; (c) após secagem por spray dry. 
Comparando a Figura 3a (antes fibrilação) e a Figura 3b (após fibrilação) observa-se que a moagem por fricção ultrafina promove a fibrilação das fibras de celulose, verificado pela diminuição de seu diâmetro, originalmente em torno de $50 \mu \mathrm{m}$. Após o processamento o diâmetro da fibra ficou em valores próximos a $50 \mathrm{~nm}$, caracterizando uma NFC, porém não foi possível medir seu comprimento uma vez que a celulose de Pinus se caracteriza por possuir fibras longas. O processo de secagem por aspersão com os parâmetros utilizados promoveu a aglomeração das NFC (Figura 3c) formando aglomerados com diâmetro médio de $8,63 \mu \mathrm{m}$, entre valores mínimos e máximos de 4,07 $\mu \mathrm{m}$ e $17,42 \mu \mathrm{m}$, respectivamente. Os grandes aglomerados não foram considerados no cálculo do diâmetro. Segundo a literatura, o material submetido a este processo de secagem tende a ter o diâmetro da gotícula produzida pelo atomizador ${ }^{[30]}$.

A Figura 4 mostra a obtenção de nanofibras de celulose, confirmando os resultados obtidos no ensaio de MEV. Observa-se que existem zonas de maiores concentrações de fibras evidenciando sua tendência a aglomerar. Pela análise da imagem observam-se fibras com comprimentos superiores à própria micrografia enquanto que o diâmetro está compreendido entre 10 e $60 \mathrm{~nm}$.

A morfologia das espumas e compósitos poliuretânicos é diretamente influenciada pela adição de cargas. Na Figura 5 pode-se observar a morfologia das amostras EPU, EPU0,5, EPU1 e EPU2. A imagem de MEV da amostra EPU indica a obtenção de células levemente heterogêneas e a adição da celulose mantém a heterogeneidade nas células da espuma, no entanto, as células tornam-se mais irregulares com o aumento da carga, com acentuada deformação na amostra EPU2. As membranas dos poros das células nas amostras EPU0,5 e EPU1 encontram-se mais rompidas enquanto que nas amostras EPU e EPU2 mantiveram-se mais intactas. Observando as células individualmente verifica-se que existe uma maior quantidade de poros nas células das espumas com cargas do que na amostra EPU. Segundo a literatura, cargas em espumas de PU afetam as paredes de
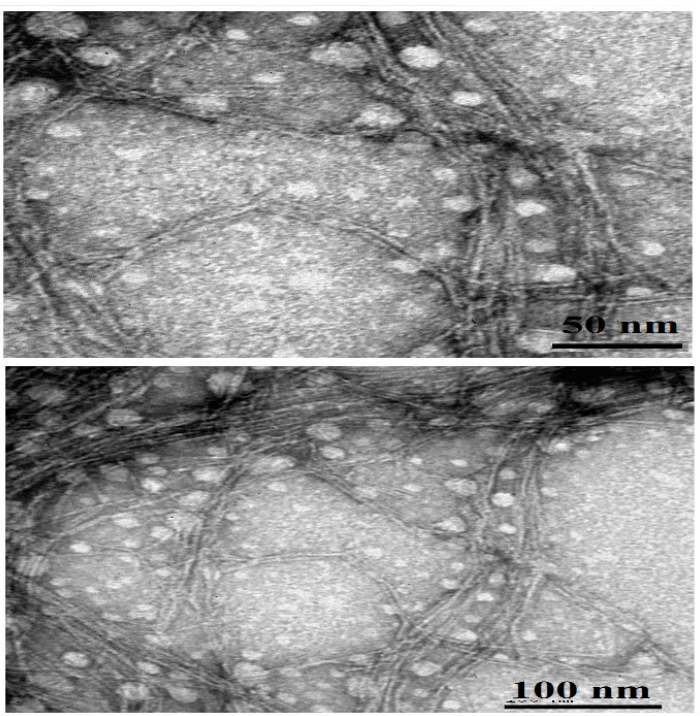

Figura 4. Micrografia de MET da amostra de celulose de Pinus após moagem.
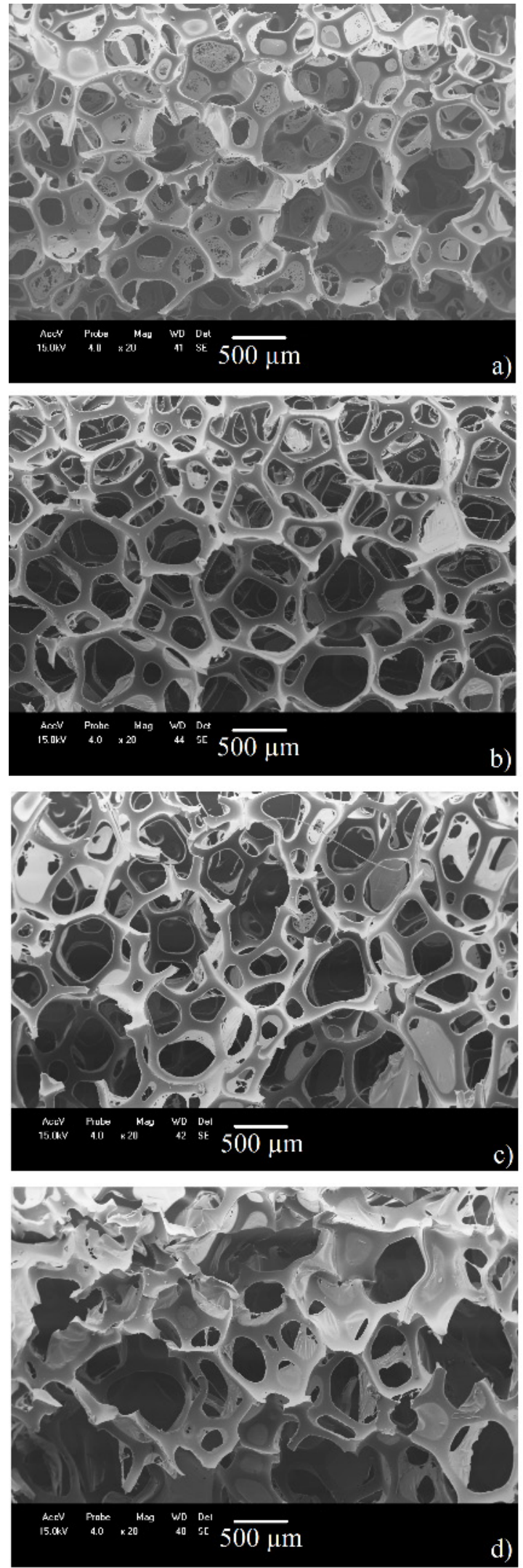

Figura 5. Micrografia de MEV da espuma e compósitos expandidos: (a) EPU; (b) EPU0,5; (c) EPU1 e (d) EPU2. 
suas células, contribuindo para ruptura de sua parede devido ao efeito desestabilizador da carga através de mecanismos de retração das arestas das células ${ }^{[1,33-35]}$. Este fato justifica a morfologia encontrada para as amostras EPU0,5 e EPU1.

Como a morfologia do material vegetal após tratamento de secagem apresentou características esféricas devido à aglomeração de NFC, esta pode ser considerada como partícula. As partículas celulósicas atuam como sítios de nucleação, diminuindo a energia necessária para criar células. Desta forma um número maior de células começa a nuclear ao mesmo tempo e, como existe menos gás disponível para seu crescimento, ocorre uma diminuição do tamanho da célula com uma maior pressão interna que pode provocar a sua coalescência (quando ocorre o rompimento da parede celular e duas células passam a ser uma única $)^{[4,35]}$. Com a amostra EPU2, como a adição de carga afeta as paredes das espumas tornando-as mais finas, pode ter ocorrido a coalescência de suas células provocando sua deformação acentuada, acarretando em uma maior quantidade de "buracos".

Pela análise da Tabela 2 observa-se que a adição de celulose em uma formulação de espuma poliuretânica acarreta em aumento no valor da densidade aparente, e o aumento da concentração de carga contribui para esta tendência, no entanto, isto não é observado para a amostra EPU2. Durante a obtenção das espumas foi observado um aumento da viscosidade devido a maior dificuldade de agitação da mistura. Nas espumas de poliuretano, a adição de fibras ao poliol promoveu um aumento da viscosidade do meio reacional, contribuindo para a diminuição da reatividade dos reagentes e impedindo a sua expansão $0^{[10,13,33]}$.

O valor da densidade de células aumenta com a adição da celulose evidenciando uma diminuição na expansão da espuma em relação ao análogo puro. Estes resultados estão de acordo com a literatura e justificam os valores de densidade aparente encontrados, mostrando que espumas com menor expansão possuem valores de densidade aparente maiore ${ }^{[1,13,35]}$. A densidade aparente da amostra EPU2 pode ser justificada pela densidade de células, pois os valores encontrados estão muito próximos aos obtidos na amostra EPU0,5. Conforme comentado anteriormente, pode ter havido coalescência das células desta amostra. Observa-se que a adição das cargas aumenta o diâmetro dos poros das espumas, embora este aumento não seja apreciável considerando a margem de erro envolvida.

Os resultados obtidos com a análise da resistência à compressão dos compósitos expandidos, segundo norma ASTM D 3574, encontram-se na Tabela 3.

Pelos resultados obtidos na Tabela 3 é possível observar que, em todos os compósitos expandidos, a adição de celulose aumenta a resistência à compressão do compósito, evidenciado por uma maior tensão necessária para realizar uma compressão de $50 \%$ em relação ao tamanho inicial da espuma. Nos compósitos EPU0,5 e EPU1 ocorreu um aumento de 40 e $50 \%$, respectivamente, na resistência à compressão em relação ao seu análogo puro. A amostra EPU2, no entanto, apresentou um aumento de $23 \%$.

A Figura 6 mostra a curva de deformação das espumas, obtida pela compressão unidirecional de uma amostra cúbica representando o comportamento mecânico quanto à resistência à compressão. É possível observar em todas
Tabela 2. Valores de densidade aparente, densidade de células e diâmetro para as espumas de PU.

\begin{tabular}{|c|c|c|c|}
\hline Amostra & $\begin{array}{c}\text { Densidade } \\
\text { aparente } \\
\left(\text { kg.m } \mathbf{m}^{-3}\right) \\
\end{array}$ & $\begin{array}{c}\text { Densidade de } \\
\text { células }\left(10^{6} \text {.cel. }\right. \\
\left.\mathbf{c m}^{-3}\right)\end{array}$ & $\begin{array}{c}\text { Diâmetro Feret } \\
\qquad(\mu \mathrm{m})\end{array}$ \\
\hline EPU & $18,67 \pm 0,67$ & $8,27 \pm 0,92$ & $341 \pm 90$ \\
\hline EPU0,5 & $23,93 \pm 1,48$ & $9,68 \pm 1,70$ & $430 \pm 145$ \\
\hline EPU1 & $26,81 \pm 3,82$ & $12,78 \pm 0,66$ & $390 \pm 142$ \\
\hline EPU2 & $23,66 \pm 1,06$ & $9,27 \pm 0,84$ & $453 \pm 163$ \\
\hline
\end{tabular}

Tabela 3. Resultados obtidos no ensaio de resistência à compressão (ASTM D-3574.).

\begin{tabular}{lcc}
\hline Material & $\begin{array}{c}\text { Resistência à } \\
\text { compressão* }(\mathbf{k P a})\end{array}$ & $\begin{array}{c}\text { Módulo de elasticidade } \\
\mathbf{( k P a )}\end{array}$ \\
\hline EPU & $3,0 \pm 0,1$ & $36,5 \pm 2,1$ \\
EPU0,5 & $4,2 \pm 0,8$ & $37,9 \pm 2,6$ \\
EPU1 & $4,5 \pm 0,6$ & $35,4 \pm 7,0$ \\
EPU2 & $3,7 \pm 0,1$ & $37,8 \pm 0,6$ \\
\hline
\end{tabular}

*Resistência à compressão a uma deformação de $50 \%$ em relação à espessura original do corpo de prova.

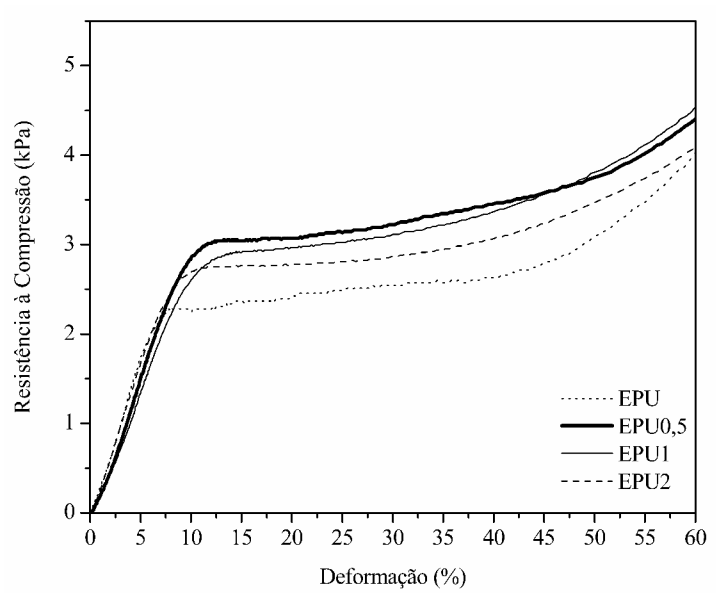

Figura 6. Curvas de resistência à compressão unidirecional de amostras cúbicas das EPU, EPU0,5; EPU1 e EPU2.

as curvas os regimes de deformação característicos de uma espuma de PU. O primeiro regime é uma região elástica linear na qual a energia de deformação inicia a deformação da célula. Na sequência observa-se uma região de plateau, no qual pequenos aumentos da carga compressiva aplicada resultam em grandes deformações das paredes das células, e a terceira região é caracterizada pela deformação total da célula com o encontro de suas paredes com as paredes de células vizinhas ocasionando aumento acentuado na resistência à compressão. Após a retirada da carga compressiva, o material retorna a sua dimensão original (espumas flexíveis) ${ }^{[8,36,37]}$.

Analisando as curvas, a amostra EPU possui o fim de sua zona elástica em torno de $7 \%(2,19 \mathrm{kPa})$, enquanto que as amostras com fibras vegetais o final da zona elástica encontra-se em aproximadamente 13\% (3,04 kPa) para EPU0,5; 15\% (2,92 kPa) para EPU1 e 13\% (2,74 kPa) para EPU2. No entanto, como mostra a Tabela 3, o módulo de elasticidade das amostras não varia, o que indica que 
as amostras possuem a mesma rigidez. O intervalo de deformação referente à zona de plateau sofre uma diminuição nas amostras com carga. Nesse sentido pode ser dito que as cargas adicionadas contribuíram para uma maior resistência das paredes das espumas após a transição do regime elástico para o plateau, pois é necessário proporcionar um maior aumento da carga compressiva para ocasionar aumento na deformação do material. Observa-se que a amostra EPU2 obteve uma redução da resistência à compressão e da densidade em relação às demais amostras com a adição de carga. Como ambas as propriedades são dependentes da estrutura e morfologia da espuma, os resultados podem ser explicados pela micrografia da Figura 5d, a qual revela uma distribuição irregular das células desta amostra, com a presença de células deformadas e de "buracos" que acabaram reduzindo suas propriedades.

\section{Conclusões}

Com o processo de fibrilação mecânica foi possível a obtenção de celulose Pinus Ellioti na forma nanofibrilada, com o diâmetro da fibra variando entre 10 e $60 \mathrm{~nm}$, suspensas em solução aquosa que, após a secagem por spray, formou microparticulados de celulose. Espumas flexíveis de PU foram obtidas com a adição de celulose, proporcionando um aumento na densidade aparente e espumas com células menores e com paredes de células mais finas. A adição de 1\% de celulose aumentou a resistência à compressão em $50 \%$ em relação ao PU puro. Porém, em quantidades superiores a $1 \%$ de celulose ocorre a redução da densidade aparente e da resistência à compressão. Assim, em quantidades adequadas, a celulose de Pinus pode ser considerada um possível aditivo para espumas flexíveis de PU em substituição aos aditivos produzidos por fontes não renováveis.

\section{Agradecimentos}

Os autores agradecem às empresas Diletto e Trombini S/A pelo fornecimento do material utilizado. Ao Centro de Microscopia e ao Centro de Desenvolvimento Tecnológico Farmacêutico da Universidade Federal do Rio Grande do Sul pelo auxílio na utilização e disponibilidade dos equipamentos e à FAPERGS pelo apoio financeiro.

\section{Referências}

1. Madaleno, L., Pyrz, R., Crosky, A., Jensen, L. R., Rauhe, J. C. M., Dolomanova, V., Timmons, A. M. M. V. B., Pinto, J. J. C., \& Norman, J. (2013). Processing and characterization of polyurethane nanocomposite foam reinforced with montmorillonite-carbon nanotube hybrids. Composites. Part A, Applied Science and Manufacturing, 44, 1-7. http://dx.doi. org/10.1016/j.compositesa.2012.08.015.

2. Çelebi, S., \& Küçük, H. (2012). Acoustic properties of tealeaf fiber mixed polyurethane composites. Cellular Polymers, 31(5), 241-255.

3. Seydibeyoğlu, M. Ö., \& Oksman, K. (2008). Novel nanocomposites based on polyurethane and micro fibrillated cellulose. Composites Science and Technology, 68(3-4), 908914. http://dx.doi.org/10.1016/j.compscitech.2007.08.008.

4. Bahrambeygi, H., Rabbi, A., Nasouri, K., Shoushtari,A. M., \& Babaei, M. R. (2013). Morphological and structural developments in nanoparticles/polyurethane foam nanocomposite's synthesis and their effects on mechanical properties. Advances in Polymer Technology, 32(S1), E545-E555. http://dx.doi.org/10.1002/ adv. 21300

5. Nikje, M. M. A., Moghaddam, S. T., Noruzian, M., Nejad, M. A. F., Shabani, K., Haghshenas, M., \& Shakhesi, S. (2013). Preparation and characterization of flexible polyurethane foam nanocomposites reinforced by magnetic core-shell Fe3O4@ APTS nanoparticles. Colloid \& Polymer Science, 292(3), 627-633. http://dx.doi.org/10.1007/s00396-013-3099-2.

6. Mishra, D., \& Sinha, V. K. (2013). Polyurethane foams from cellulosic waste and natural oil-based polyols: a modified approach. Polymers from Renewable Resources, 4(2), 85-107.

7. Navarro, M. V., Vega-Baudrit, J. R., Sibaja, M. R., \& Melero, F. J. (2012). Use of rice husk as filler in flexible polyurethane foams. Macromolecular Symposia, 321-322(1), 202-207. http:// dx.doi.org/10.1002/masy.201251136.

8. Elliott, J. A., Windle, A. H., Hobdell, J. R., Eeckhaut, G., Oldman, R. J., Ludwig, W., Boller, E., Cloetens, P., \& Baruchel, J. (2002). In-situ deformation of an open-cell flexible polyurethane foam cgaracterised by $3 \mathrm{D}$ computed microtomography. Journal of Materials Science, 37(8), 1547 1555. http://dx.doi.org/10.1023/A:1014920902712.

9. Wolska, A., Goździkiewicz, M., \& Ryszkowska, J. (2012). Influence of graphite and wood-based fillers on the flammability of flexible polyurethane foams. Journal of Materials Science, 47(15), 5693-5700. http://dx.doi.org/10.1007/s10853-0126394-2.

10. Yuan, J., \& Shi, S. (2009). Effect of the addition of wood flours on the properties of rigid polyurethane foam. Journal of Applied Polymer Science, 113(5), 2902-2909. http://dx.doi. org/10.1002/app.30322.

11. Mello, D., Pezzin, S. H., \& Amico, S. C. (2009). The effect of post-consumer PET particles on the performance of flexible polyurethane foams. Polymer Testing, 28(7), 702-708. http:// dx.doi.org/10.1016/j.polymertesting.2009.05.014.

12. Zimmermann, M. V. G., Turella, T. C., \& Zattera, A. J. (2014). Influência do tratamento químico da fibra de bananeira em compósitos de poli(etileno-co-acetato de vinila) com e sem agente de expansão. Polímeros: Ciência e Tecnologia, 24(1), 58-64. http://dx.doi.org/10.4322/polimeros.2013.071.

13. Silva, M. C., Takahashi, J. A., Chaussy, D., Belgacem, M. N., \& Silva, G. G. (2010). Composites of rigid polyurethane foam and cellulose fiber residue. Journal of Applied Polymer Science, 117(6), 3665-3672.

14. Fornasieri, M., Alves, J. W., Muniz, E. C., Ruvolo-filho, A., Otaguro, H., Rubira, A. F., \& Carvalho, G. M. (2011). Synthesis and characterization of polyurethane composites of wood waste and polyols from chemically recycled pet. Composites. Part A, Applied Science and Manufacturing, 42(2), 189-195. http:// dx.doi.org/10.1016/j.compositesa.2010.11.004.

15. Chen, X., Yuc, J., Zhang, Z., \& Lu, C. (2011). Study on structure and thermal stability properties of cellulose fibers from rice straw. Carbohydrate Polymers, 85(1), 245-250. http://dx.doi. org/10.1016/j.carbpol.2011.02.022.

16. Khalil, H. P. S. A., Davoudpour, Y., Islam, M. N., Mustapha, A., Sudesh, K., Dungani, R., \& Jawaid, M. (2014). Production and modification of nanofibrillated cellulose using various mechanical processes: A review. Carbohydrate Polymers, 99, 649-665. PMid:24274556. http://dx.doi.org/10.1016/j. carbpol.2013.08.069.

17. Abe, K., \& Yano, H. (2011). Formation of hydrogels from cellulose nanofibers. Carbohydrate Polymers, 85(4), 733-737. http://dx.doi.org/10.1016/j.carbpol.2011.03.028.

18. Jonoobi, M., Mathew, A. P., \& Oksman, K. (2012). Producing low-cost cellulose nanofiber from sludge as new source of raw 
materials. Industrial Crops and Products, 40, 232-238. http:// dx.doi.org/10.1016/j.indcrop.2012.03.018.

19. Lavoine, N., Desloges, I., Dufresne, A., \& Bras, J. (2012). Microfibrillated cellulose: its barrier properties and applications in cellulosic materials: a review. Carbohydrate Polymers, 90(2), 735-764. PMid:22839998. http://dx.doi.org/10.1016/j. carbpol.2012.05.026.

20. Missoum, K., Martoïa, F., Belgacem, M. N., \& Bras, J. (2013). Effect of chemically modified nanofibrillated cellulose addition on the properties of fiber-based materials. Industrial Crops and Products, 48, 98-105. http://dx.doi.org/10.1016/j. indcrop.2013.04.013.

21. Siró, I., \& Plackett, D. (2010). Microfibrillated cellulose and new nanocomposite materials: a review. Cellulose (London, England), 17(3), 459-494. http://dx.doi.org/10.1007/s10570010-9405-y.

22. Afra, E., Yousefi, H., Hadilam, M. M., \& Nishino, T. (2013). Comparative effect of mechanical beating and nanofibrillation of cellulose on paper properties made from bagasse and softwood pulps. Carbohydrate Polymers, 97(2), 725-730. PMid:23911507. http://dx.doi.org/10.1016/j.carbpol.2013.05.032.

23. Floros, M., Hojabri, L., Abraham, E., Jose, J., Thomas, S., Pothan, L., Leao, A. L., \& Narine, S. (2012). Enhancement of thermal stability, strength and extensibility of lipid-based polyurethanes with cellulose-based nanofibers. Polymer Degradation \& Stability, 97(10), 1970-1978. http://dx.doi. org/10.1016/j.polymdegradstab.2012.02.016.

24. Klemm, D., Kramer, F., Moritz, S., Lindström, T., Ankerfors, M., Gray, D., \& Dorris, A. (2011). Nanocelluloses: a new family of nature-based materials. Angewandte Chemie, 50(24), 5438-5466. PMid:21598362. http://dx.doi.org/10.1002/ anie. 201001273 .

25. Yousefi, H., Nishino, T., Shakeri, A., Faezipour, M., Ebrahimi, G., \& Kotera, M. (2013). Water-repellent all-cellulose nanocomposite using silane coupling treatment. Journal of Adhesion Science and Technology, 27(12), 1324-1334. http:// dx.doi.org/10.1080/01694243.2012.695954.

26. Peng, Y., Han, Y., \& Gardner, D. J. (2012). Spray-drying cellulose nanofibrils: effect of drying parameters on particle morphology and size distribuition. Wood and Fiber Science, 44(4), 1-14.

27. Amin, M. C. I. M., Abadi, A. G. A., \& Katas, H. (2014). Purification, characterization and comparative studies of spray-dried bacterial cellulose microparticles. Carbohydrate Polymers, 99(2), 180-189. PMid:24274495. http://dx.doi. org/10.1016/j.carbpol.2013.08.041.

28. Vartiainen, J., Pöhler, T., Sirola, K., Pylkkänen, L., Alenius, H., Hokkinen, J., Tapper, U., Lahtinen, P., Kapanen, A., Putkisto, K., Hiekkataipale, P., Eronen, P., Ruokolainen, J., \& Laukkanen, A. (2011). Health and environmental safety aspects of friction grinding and spray drying of microfibrillated cellulose. Cellulose, 5(10), 775-786. http://dx.doi.org/10.1007/ s10570-011-9501-7.

29. Paude, L. A., Worku, Z. A., Meeus, J., Guns, S., \& Mooter, G. V. D. (2013). Manufacturing of solid dispersions of poorly water soluble drugs by spray drying: Formulation and process considerations. International Journal of Pharmaceutics, 453(1), 253-284. PMid:22820134. http://dx.doi.org/10.1016/j. ijpharm.2012.07.015.

30. Oliveira, O. W., \& Petrovick, P. R. (2010). Secagem por aspersão (spray drying) de extratos vegetais: bases e aplicações. Brazilian Journal of Pharmacognosy, 20(4), 641-650. http:// dx.doi.org/10.1590/S0102-695X2010000400026.

31. Verdolotti, L., Salerno, A., Lamanna, R., Nunziata, A., Netti, P., \& Iannace, S. (2012). A novel hybrid PU-alumina flexible foam with superior hydrophilicity and adsorption of carcinogenic compounds from tobacco smoke. Microporous and Mesoporous Materials, 151, 79-87. http://dx.doi.org/10.1016/j. micromeso.2011.11.010

32. Jahanmardi, R., Kangarlou, B., \& Dibazar, A. R. (2013). Effects of organically modified nanoclay on cellular morphology, tensile properties, and dimensional stability of flexible polyurethane foams. Journal of Nanostructure in Chemistry, 3(82), 1-6. http://dx.doi.org/10.1186/2193-8865-3-82.

33. Gu, R., Sain, M. M., \& Konar, S. K. (2013). A feasibility study of polyurethane composite foam with added hardwood pulp. Industrial Crops and Products, 42, 273-279. http://dx.doi. org/10.1016/j.indcrop.2012.06.006.

34. Bernal, M. M., Lopez-Manchado, M. A., \& Verdejo, R. (2011). In situ foaming evolution of flexible polyurethane foam nanocomposites. Macromolecular Chemistry and Physics, 212(9), 971-979. http://dx.doi.org/10.1002/macp.201000748.

35. Silva, V. R., Mosiewicki, M. A., Yoshida, M. I., Silva, M. C., Stefani, P. M., \& Marcovich, N. E. (2013). Polyurethane foams based on modified tung oil and reinforced with rice husk ash I: synthesis and physical chemical characterization. Polymer Testing, 32(2), 438-445. http://dx.doi.org/10.1016/j. polymertesting.2013.01.002.

36. Jankowski, M., \& Kotelko, M. (2010). Dynamic compression tests of a polyurethane flexible foam as a step in modelling impact of the head to the vehicle seat head restrain. FME Transactions, 38, 121-127.

37. Avalle, M., Belingardi, G., \& Montanini, R. (2001). Characterization of polymeric structural foams under compressive impact loading by means of energy-absorption diagram. International Journal of Impact Engineering, 25(5), 455-472. http://dx.doi. org/10.1016/S0734-743X(00)00060-9.

Enviado: Maio 19, 2015

Revisado: Nov. 06, 2015

Aceito: Jan. 28, 2016 